\title{
Engagement transnational des descendants d'immigrés : carrières militantes et « rapport aux origines »
}

The Transnational Engagement of the Descendants of Immigrants: Activist

Careers and "Ties to Origins"

\section{Laurent Lardeux}

\section{(2) OpenEdition Journals}

Édition électronique

URL : http://journals.openedition.org/conflits/19881

DOI : 10.4000/conflits.19881

ISSN : $1777-5345$

Éditeur :

CCLS - Centre d'études sur les conflits lilberté et sécurité, L'Harmattan

Édition imprimée

Date de publication : 20 juillet 2018

Pagination : 61-82

ISBN : 978-2-343-15504-3

ISSN : $1157-996 X$

\section{Référence électronique}

Laurent Lardeux, « Engagement transnational des descendants d'immigrés : carrières militantes et " rapport aux origines » », Cultures \& Conflits [En ligne], 109 | printemps 2018, mis en ligne le 20 juillet 2020, consulté le 30 mars 2021. URL : http://journals.openedition.org/conflits/19881 ; DOI : https:// doi.org/10.4000/conflits. 19881 


\title{
Engagement transnational des descendants d'immigrés : carrières militantes et « rapport aux origines»
}

\section{Laurent LARDEUX}

Laurent Lardenx est chargé d'études et de recherche à l'Institut national de la jeunesse et de l'éducation populaire (INJEP) et chercheur associé an laboratoire Triangle (ENS de Lyon / UMR 5206). Après une thèse menée à l'Université Lyon 2 sur la question des rapatriements de réfugiés en Afrique centrale, il a engagé à l'INJEP des travaux sur l'engagement, les dispositifs de participation ou encore les activités politiques transnationales des jeunes issus de l'immigration. Il a rédigé plusieurs livres, articles et chapitres d'ouvrage sur ces questions, dont Retours d'exil. Ethnographie des rapatriements de réfugiés, Éditions de l'EHESS, 2015; " "Libre consentement" au retour des réfugiés congolais (RDC) et nouvelles normes d'application du rapatriement par le HCR ", Critique internationale, $n^{\circ}$ 56, 2012, pp. 95-116; "Collectifs cosmopolitiques de réfugiés urbains en Afrique centrale. Entre droits de l'homme et "droit de cité" ", Revue française de science politique, vol. 59, $n^{\circ} 4$, 2010, pp. 783-804.

\begin{abstract}
Asocier la notion d'engagement transnational à celle de descendants d'imA migrés ne va pas forcément de soi et génère bien souvent des séries d'imageries antagonistes qui laissent peu de place à la nuance. Deux représentations radicalement différentes se sont cristallisées ces dernières années autour de deux événements médiatiques majeurs, emblématiques tour à tour des craintes ou de l'angélisme que peuvent susciter des formes d'engagement orientées audelà du cadre strict des États-nations.
\end{abstract}

Revenons d'abord au printemps 2011, et à ce qui fut rapidement dénommé le Printemps arabe. Alors que le monde suit en direct les soulèvements en Tunisie, en Egypte, en Syrie, ou encore en Libye, les premiers reportages mettent la lumière sur les «passeurs d'images » des révolutions. On découvre en Europe une jeunesse mobilisée dans des actions militantes sur des 
espaces qui débordent les frontières nationales du pays de naissance ou de résidence ${ }^{1}$, on salue les actions citoyennes et le nouvel élan démocratique de ces descendants de migrants engagés pour défendre des causes politiques dans leur pays d'origine. Ces soulèvements ont aussi transformé les représentations portées sur l'engagement militant des descendants d'immigrés présents en Europe avec l'éclosion, les mois qui ont suivi les soulèvements, d'associations, collectifs, organisations à travers lesquels se sont reconstruits des actions de solidarité, des liens et des réseaux militants au niveau local et transnational 2. Que reste-t-il de ces représentations en 2017 ? Six ans plus tard, avec la série d'attentats qui touche l'Europe, l'espace médiatique et politique véhicule une toute autre imagerie de ces descendants de migrants engagés hors des cadres nationaux. Il n'est plus question d' « engagement transnational » mais de « radicalisation "; l'idéalisation romantique du jeune démocrate révolutionnaire qui avait submergé l'espace public a cédé sa place à celle du « djihadiste fondamentaliste ». On souligne la supposée perte de valeurs républicaines d'une partie toujours plus grande de la jeunesse populaire, qui sans être véritablement nommée, concerne en priorité des jeunes issus de l'immigration postcoloniale subsaharienne et maghrébine ${ }^{3}$.

Ces deux évènements, aussi paroxystiques soient-ils, n’en révèlent pas moins la persistance de lignes de clivage entre deux approches de l'engagement transnational autour desquelles se nouent des échanges contradictoires : celle tournée vers l'intégration dans le pays de résidence et l'autre concernée par les questions du politique, du religieux et/ou du développement dans les régions d'origine. Ces perspectives, dichotomiques et cloisonnantes, tendent par ailleurs à négliger les processus de transmission d'une mémoire des origines dans le cadre de pratiques d'engagement transnationales. En opposant « intégration ici » et « développement là-bas », les approches habituellement mobilisées ne saisissent souvent pas les modalités de réappropriation de l'histoire familiale par les descendants d'immigrants et rendent insuffisamment compte du rôle central joué par les associations ou collectifs dans la conversion de la mémoire en modalité d'action ${ }^{4}$. Au croisement de la sociologie politique et de

1. Sur le rôle des descendants d'immigrés pendant les révolutions arabes, voir Geisser V., "Quelle révolution pour les binationaux ? Le rôle des Franco-Tunisiens dans la chute de la dictature et dans la transition politique », Migrations Société, n¹43, 2012, pp. 155-178; Labat S., «Les binationaux franco-algériens : un nouveau rapport entre nationalité et territorialité », Critique internationale, ${ }^{\circ} 56,2012$, pp. 77-94.

2. Jaulin T., Nilsson B., "Voter ici et là-bas : les Tunisiens à l'étranger depuis 2011 ", Revue européenne des migrations internationales, n³-4, 2015, pp. 41-71.

3. Boubeker A., "Les mondes de l'immigration des héritiers. Ancrages et transmigration », Multitudes, $\mathrm{n}^{\circ} 49,2012$, pp. 100-110.

4. Bien que rares, quelques travaux ont récemment été entrepris sur la question de l'engagement transnational des descendants d'immigrés. Signalons notamment les recherches de Monika Hess et Benedikt Korf sur les espaces transnationaux de l'engagement, en Suisse, de Tamouls sri-lankais de la seconde génération pendant et après l'assaut final de l'armée sri-lankaise. Ces espaces politiques de l'activisme sont à concevoir à l'intersection des évènements au Sri Lanka et de la situation passive ou indifférente de la société hôte sur ces évènements. Hess M., Korf 
la sociologie pragmatique, notre recherche se donne précisément pour objectif d'observer les carrières d'engagement transnational des jeunes descendants de migrants à partir des systèmes d'articulation entre d'une part, les évènements macrosociaux survenus dans l'espace d'origine (transition, soulèvement, vie démocratique, etc.) et, d'autre part, les changements microsociaux survenus dans les biographies entendus en termes de « bifurcation ${ }^{5}$ » ou de « réversibilité $6 »$. Comment ces croisements entre niveau structurel et niveau personnel, entre contexte macrosocial et cadre microsocial d'interprétation et de pratiques militantes modifient-ils le sens des trajectoires d'engagement et les différentes formes de citoyenneté développées ensuite par ces jeunes dans des horizons d'action à la fois locaux, nationaux et transnationaux ?

À partir de quelques exemples significatifs de «carrières militantes transnationales » de jeunes issus de l'immigration ( $c f$. encadré), notre propos vise à articuler le temps court de l'engagement transnational au temps long de l'histoire familiale. La construction des carrières militantes transnationales est ainsi analysée en amont, c'est-à-dire au niveau des expériences de l'immigration potentiellement génératrices de ressources, et en aval au niveau des fenêtres d'opportunités qui peuvent s'ouvrir dans le cours des évènements politiques, sociaux, culturels en France et dans le pays d'origine. L'enjeu n'est donc pas de penser la subjectivité de l'engagement et l'objectivité d'une cause comme deux réalités disjointes, mais de se situer au plus près de l'expérience des jeunes engagés et dans la succession des espaces traversés.

À partir de trente-cinq entretiens biographiques réalisés entre juillet 2015 et mai 2016 auprès de jeunes descendants de migrants engagés dans différents types d'associations transnationales, nous rendrons compte dans une première partie des éléments déclencheurs intervenant dans l'activation des carrières militantes transnationales. Que ce soit par l'ajustement des actions individuelles à celles de leurs partenaires ou par la justification de la cause défendue pour la rendre légitime aux yeux de l'opinion publique, ce travail de mise en forme restitué au cours des entretiens biographiques se structure autour de deux pôles : celui de la revendication des appartenances transnationales comme acte de reconnaissance d'une altérité qu'il convient de protéger dans

B., «Tamil diaspora and the political spaces of second generation activism in Switzerland ", Global Networks, n¹4, 2014, p. 419-437.

5. Selon Marc Bessin, Claire Bidart et Michel Grossetti, on peut qualifier de bifurcation «t toute situation sociale dans laquelle un évènement imprévisible a des effets irréversibles, c'est-à-dire durables, et qui, amorcé dans un contexte spécifique, a des conséquences sur des registres plus étendus de la vie sociale » (Grossetti M., Bessin M. et C. Bidart, Bifurcations, Paris, La Découverte, 2009, p. 7).

6. Les discontinuités biographiques et la diversité des turning-points dans les carrières des migrants traduisent un processus d'individuation fait d'incertitudes et de réversibilités de positions, de places et de situations (Roulleau-berger L., « Repenser la question migratoire : migrations, inégalités multisituées et individuation ». Sociologies [En ligne], Dossier, Migrations, pluralisation, ethnicisation des sociétés contemporaines, mis en ligne le 18 octobre 2011, URL : http://journals.openedition.org/sociologies/3701, consulté le 18 mai 2018). 
l'espace du quartier, et celui de l'affirmation d'une citoyenneté cumulative comme acte de résistance aux discriminations auxquelles les descendants de migrants tentent de faire face. Par-delà ces deux pôles et les interactions qu'ils induisent entre les échelles globales, locales et " glocales », ce sont les différentes modalités de passage des pratiques de citoyenneté d'une génération immigrée à une autre issue de l'immigration qu'il nous semble essentiel de souligner dans une seconde partie. Il s'agit plus spécifiquement de voir comment le renouvellement des générations contribue aussi à modifier les horizons d'action et les formes d'appartenance locale, nationale et transnationale.

\section{Le matériau d'enquête}

Cette recherche, réalisée dans le cadre d'un rapport de l'INJEP 7 s'appuie sur un matériel empirique recueilli à partir de trente-cinq entretiens biographiques réalisés entre juillet 2015 et mai 2016 dans quatre villes de la région Ile-de-France où se concentrent des grands ensembles habités majoritairement par des populations immigrées et issues de l'immigration $d^{\prime}$ Afrique du Nord et d'Afrique subsaharienne ${ }^{8}$ : Aubervilliers, Saint-Denis, Vitry-sur-Seine, Ivry.

Dans notre enquête, nous avons retenu comme définition des Français de 16-35 ans nés en France ou ayant rejoint la France à un âge très jeune, soit avant la scolarisation obligatoire, et dont le père et/ou la mère est/sont immigré.e.s. En raison de la diversité des organisations vers lesquelles ces jeunes s'engagent, mais aussi des profils d'individus amenés à participer aux activités des différents collectifs ou structures associatives, il importait de reproduire au mieux cette pluralité, du point de vue du sexe (18 femmes et 17 hommes), du niveau d'études (16 jeunes diplômés du supérieur ou lycéens poursuivant leurs études en section générale, 19 ayant terminé leurs études avant le bac) et de l'ancienneté de l'engagement (16 engagés dans un collectif depuis plus de trois ans, 19 depuis moins de trois ans). Nous avons enfin retenu dans notre échantillon des jeunes issus de l'immigration d'Afrique du Nord et d'Afrique subsaharienne (13 originaires du Maghreb, 10 d'Afrique de l'Ouest, 12 d'Afrique centrale) en raison, en premier lieu, de leur importance démographique dans les quartiers enquêtés et, en second lieu, dans l'objectif de questionner le rapport à l'histoire coloniale d'une génération immigrée à une autre issue de l'immigration.

Face à la constellation de groupements associatifs possibles, trois types d'organisations citoyennes transnationales ont été retenues dans notre étude :

- des structures fortement politisées, apparentées à des partis d'opposition en exil ;

- des mouvements humanistes, avec ou sans affiliation politique, visant principalement la défense des droits de l'homme, la liberté d'opinion ou d'expression dans le pays d'origine ;

- des groupements à vocation humanitaire, dans des logiques de codéveloppement. 


\section{Les dynamiques structurelles et personnelles de l'engagement transnatio- nal}

Notre objectif est moins ici d'analyser la structuration des associations de descendants d'immigrés que de porter l'attention sur la façon dont ces derniers se positionnent vis-à-vis de ces collectifs au fur et à mesure de leurs parcours biographiques et des évolutions sociopolitiques des contextes d'installation et d'origine. La notion de « carrière militante 9 » telle que nous la mobilisons dans cette recherche permet donc de lier, plutôt que d'opposer, différentes échelles pour rendre compte des jeux d'influences réciproques, dans le temps et dans l'espace, du domaine subjectif des cadres de l'expérience contestataire 10 et du domaine objectif des «structures d'opportunités politiques ${ }^{11}$ » pouvant être fournies à certains moments de l'actualité nationale et internationale des différents groupes concernés.

Ce niveau structurel n'est bien sûr pas incompatible avec des explications d'ordre plus personnel en lien avec la trajectoire biographique de ces jeunes, ces deux dimensions, structurelle et personnelle, macro et microsociale, se trouvant au contraire fortement liées. Les parcours d'engagement sont en effet ici conçus dans cet encastrement entre dimension objective et dimension subjective, entre situation sociétale et condition personnelle. Deux perspectives

7. Pour plus de détails sur l'étude et les carrières militantes des descendants de migrants, voir Lardeux L., Engagement transnational des descendants de migrants, Rapport d'étude, INJEP, décembre 2016.

8. Le choix de privilégier les quartiers populaires se justifie par la part importante d'immigrés et de descendants d'immigrés qui y résident. Selon les données de l'Observatoire national de la politique de la ville (ONPV), il apparaît qu'un immigré sur cinq et près d'un descendant d'immigré sur sept vit dans des quartiers prioritaires de la politique de la ville (QPV), chiffre largement supérieur à celui de l'ensemble de la population, qui se situe à $7 \%$ (ONZUS, Rapport 2013, 2014 (URL : www.onpv.fr/uploads/media_items/rapport-de-l-onzus2013.original.pdf, page consultée le 18 mai 2018). Mais ces moyennes cachent des disparités très fortes. Selon l'enquête Trajectoires et Origines, la proportion est maximale pour les descendants d'immigrés subsahariens $(28 \%)$ et minimale $(6 \%)$ pour les descendants d'immigrés européens (Beauchemin C., Hamel C., Simon P., Trajectoires et origines. Enquête sur la diversité des populations en France, Ined éditions 2016, 623 p.).

9. Fillieule O., "Carrière militante », in Fillieule O., Mathieu L., Péchu C. (dir.), Dictionnaire des mouvements sociaux, Paris, Les presses de Science Po, 2009.

10. La frame perspective telle qu'elle s'est développée dans le domaine des mouvements sociaux vise, de ce point de vue, à rendre compte du travail de signification des acteurs engagés dans des mouvements sociaux. Ce travail de production oscille entre d'une part une analyse structurale des contraintes qui pèsent sur la définition des situations et sur la configuration des actions, et d'autre part une analyse interactionniste des schèmes d'action qui s'inventent dans le cours temporel des séries d'interaction. Voir Snow D. et R. D. Benford, «Ideology, Frame Resonance and Participant Mobilization ", International Social Movement Research, 1988, n¹, pp. 197-219.

11. Doug McAdam a adopté au début des années 1980 le concept de «structure des opportunités politiques ». La notion se présente à ses yeux comme la mieux adaptée pour révéler ce que la possibilité d'émergence d'une action protestataire doit, indépendamment de la capacité mobilisatrice du groupe concerné, aux évolutions conjoncturelles du système politique. Voir McAdam D., Political Process and the Development of Black Insurgency, 1930-1970, Chicago, Chicago University Press, 1982. 
peuvent ici se dégager pour saisir ces liens : l'une fait l'hypothèse d'un « transnationalisme dans l'action » qui se formerait en relation avec des événements survenus dans le pays d'origine et qui susciterait rétroactivement des aspirations à l'engagement afin de se sentir partie-prenante de l'" histoire en marche »; l'autre émet la possibilité d'un «transnationalisme en réaction » à différentes formes de discrimination subies en France : le pays d'origine est alors vu comme nouvel horizon des possibles pour des jeunes dont l'avenir en France semble fermé.

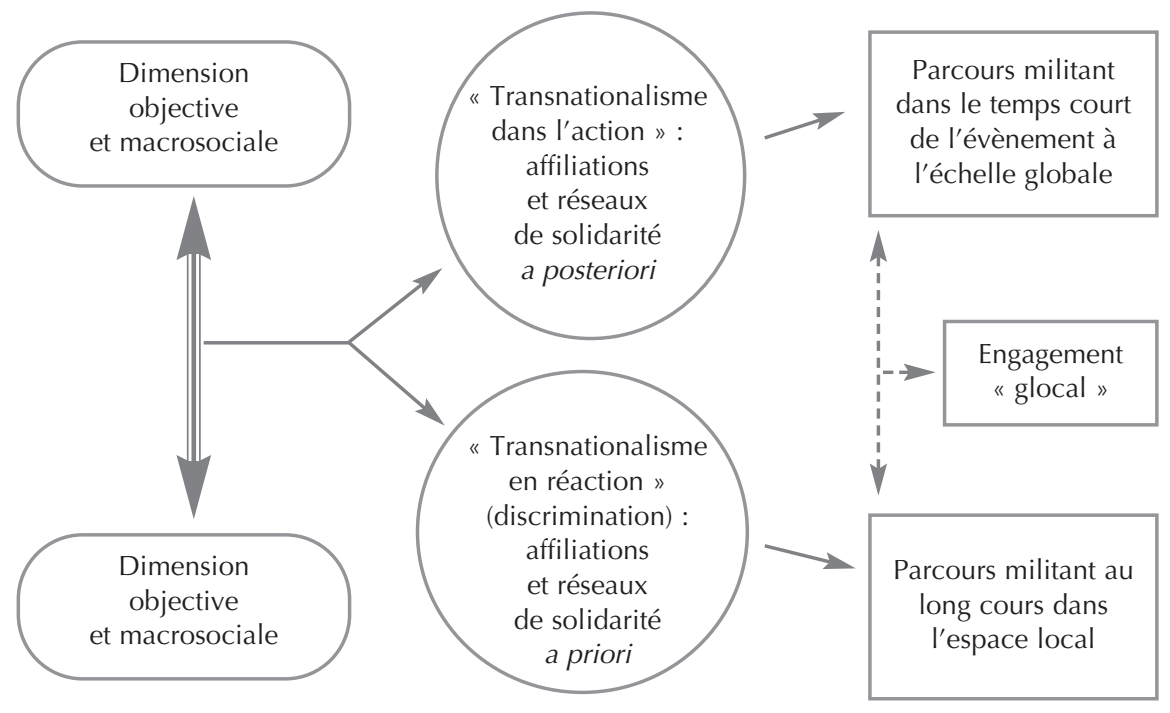

Graphique 1 : Engagement transnational " dans l'action » et " en réaction »

L'engagement transnational dans l'action : subir global et agir à partir de l'espace local

Nous souhaitons insister ici sur le rôle central joué par l'« évènement » dans les formes d'engagement de certains descendants de migrants à partir de leur territoire de vie. Certains grands moments historiques rencontrés dans le pays d'origine, qu'ils soient liés à un changement de régime, une révolution, un conflit guerrier, ou une catastrophe naturelle peuvent bouleverser les cadres interprétatifs et cognitifs des individus qui se réclament de sa citoyen- 
neté, à l'intérieur comme à l'extérieur de ses frontières. Ces évènements se croisent avec les biographies et peuvent influer en profondeur leurs cours. Cette prise en compte de la puissance socialisatrice de l'évènement en matière d'engagement transnational peut se décliner de deux façons distinctes : d'une part, des vocations militantes révélées dans le temps court de l'évènement et à partir de "solidarités a posteriori » organisées autour d'un vécu commun ; d'autre part, des carrières militantes au long cours que le contexte local contribue à retravailler et à réorienter. Ces dernières se construisent sur la base de "solidarités a priori » constituées de réseaux fermement enracinés dans la vie quotidienne (associations de migrants, partis politiques de l'étranger, associations de défense des droits de l'homme, etc.).

Dans le premier cas, propre aux vocations militantes dans le temps court de l'évènement, il s'agit de différents « groupes circonstanciels 12 » dont la spécificité réside principalement dans les effets fédérateurs qu'exerce l'événement historique dans des quartiers populaires où se concentrent des populations immigrées ou issues de l'immigration. Pour Sajida, 35 ans, salariée dans une association humanitaire et militante à Saint-Denis dans un collectif de défense des droits de l'homme, le mouvement de contestation survenu au Maroc le 20 février 2011 a été un puissant moteur d'engagement, non pas tant en raison des espoirs de transition démocratique que cet événement a pu susciter, que du fait de l'émulation produite par les sympathisants du mouvement réunis, audelà de certaines divergences, autour d'une même cause. Dans son quartier où les associations marocaines étaient déjà présentes mais relativement peu fréquentées par les descendants d'immigrés, Sajida évoque le « besoin urgent pour les Marocains, Franco-marocains, Français d'origine marocaine, première génération, seconde ou troisième génération, de participer tous ensemble à la lutte 13 ». À ses côtés, des militants investis depuis de longues années, mais aussi des jeunes issus de l'immigration qui, comme elle, découvrent de nouvelles motivations politiques à s'engager pour la transition démocratique de leur pays d'origine. Ces évènements ont été pour Sajida l'occasion de rencontrer une grande diversité de militants qui lui étaient jusqu'alors inconnus :

\footnotetext{
«C'était une plongée extraordinaire dans une communauté très particulière, de gens très militants, très politiquement engagés, que ce soit de l'extrême gauche, des syndicalistes, des droit-de-l'hommiste, mais avec qui je ne me retrouvais pas, et moi je portais un autre regard, d'autres idées, et je n'étais pas la seule (...). Ça a permis à beaucoup de gens de se réveiller, d'avoir une conscience politique ». Sajida, 35 ans, Maroc
}

12. Sur la notion de " groupes circonstanciels », voir Vilain J.-P., Lemieux C., " La mobilisation des victimes d'accidents collectifs. Vers la notion de "groupe circonstanciel” ", Politix, $\mathrm{n}^{\circ} 44$, 1998, pp. 135-160.

13. Extrait d'entretien, Sajida, 35 ans, Maroc. 
De la même manière, un certain nombre d'associations transnationales présentes dans les quartiers à forte concentration de populations immigrées et issues de l'immigration sont nées des bouleversements historiques rencontrés dans certaines régions du monde, à l'image de la démultiplication de regroupements tunisiens apparue en France à la suite du Printemps arabe ${ }^{14}$. Comme le souligne à ce sujet Daniel Cefaï, « les chocs moraux produisent une prise de conscience brutale des problèmes et conduisent à s'engager pour une cause $15 »$. Ces affiliations reposent sur des solidarités nouvelles directement organisées autour du ressenti émotionnel commun de l'évènement. La mobilisation transnationale est ainsi étroitement liée à l'irruption de l'évènement dans le cours de la vie ordinaire et diffère sensiblement des ressorts habituels de l'action collective qui implique le partage préalable d'affinités sociales ou politiques ou le soutien de réseaux d'interconnaissances déjà existants. Pour Amira, militante à Aubervilliers dans un collectif de défense des droits de l'homme en Tunisie bien avant les révolutions arabes, ces évènements ont été l'occasion de sensibiliser davantage de descendants d'immigrés qui pouvaient se sentir relativement éloignés de l'actualité politique tunisienne. En charge de la communication dans ce collectif et issue d'une famille fortement politisée, ces évènements lui ont permis de renouveler en profondeur le vivier de militants et d'aller à la rencontre d'une « jeunesse qui n'était pas du sérail » et se trouvant en forte minorité dans le collectif tunisien auquel elle appartient :

«Une bonne partie des gens qui manifestaient, qui faisaient des actions dans le cadre du Printemps arabe n'avaient jamais milité auparavant. Je dirais un tiers ou $40 \%$ des gens, c'est cet événement historique qui les a transformés. Par la suite je ne sais pas s'ils continuent à militer politiquement. Militer politiquement sur les réseaux sociaux oui, je les suis, je les connais. Mais au niveau associatif je ne sais pas, mais quand je fais appel à eux ils sont partants ».

Amira, 29 ans, Tunisie

Ces affiliations nouvelles favorisent l'affirmation d'une identité collective revendiquée publiquement. Celle-ci se fonde sur la mise en scène de solidarités de l'évènement qui semblent trouver leurs origines dans le vécu commun d'un même bouleversement historique, indépendamment des vecteurs ordi-

14. Vincent Geisser souligne à ce sujet qu' « entre janvier et juin 2011, nous avons pu recenser plus d'une centaine d'initiatives portées par les héritiers de l'immigration tunisienne en France allant de la création ex nibilo d'associations de solidarité avec la "révolution tunisienne" à la mise en place d'initiatives humanitaires pour soutenir les régions sinistrées et venir en aide aux réfugiés à la frontière tuniso-libyenne, en passant par l'organisation de colloques, séminaires, tables rondes ou le lancement de blogs racontant les exploits des révolutionnaires tunisiens » (Geisser V., "Quelle révolution pour les binationaux ? Le rôle des Franco-Tunisiens dans la chute de la dictature et dans la transition politique », art. cit., p. 170).

15. Cefaï D., «Les cadres de l'action collective : définitions et problèmes », in Cefaï D., Trom D. (dir.), Les formes de l'action collective. Mobilisations dans des arènes publiques, Paris, Éditions de l'EHESS, 2001, pp. 51-97. 
naires de la socialisation militante. Cette forme naissante d'action collective transnationale de la part de jeunes originaires de pays en phase de transition politique ou de conflit peut être interprétée en termes de rupture événementielle qui implique de profonds remaniements dans la façon de s'impliquer et de participer à la vie civique. À chaque soubresaut de l'évènement, ce sont aussi de nouvelles activations mobilisatrices qui se créent et qui contribuent aussi à durcir la frontière entre un avant et un après évènement.

Le second cas, spécifique aux « carrières militantes au long cours », caractérise davantage des situations où l'évènement, sans être déclencheur de l'acte d'engagement, vient toutefois modifier ou troubler le sens de la trajectoire militante déjà existante. Certaines situations nouvelles dans le pays d'origine peuvent ainsi contribuer à renforcer les motivations de départ, sans pour autant que les organisations n'interviennent pour susciter ou réactiver ces intentions. Des jeunes inscrits dans des collectifs militants ou des réseaux de solidarité recomposent ou réorientent leurs affiliations, à l'image de Kaïs, 33 ans, descendant d'immigrés tunisiens, dont la participation aux activités d'une association tunisienne visait dans un premier temps à participer à des collectes de fonds en faveur de projets de développement. Mais l'absence de dimension politique de l'association et l' « inertie 16 » de ses membres lors des évènements de 2011 l'ont rapidement incité à rejoindre « le cortège des progressistes 17 » selon ses termes, dans un collectif de défense des droits de l'homme fortement investi depuis le début des années 2000 sur la question de la transition démocratique. Sans être un déclencheur direct de ses activités militantes, la révolution tunisienne a activé une dimension plus politique qui n'était alors qu'au « simple état embryonnaire » en l'incitant à transformer profondément le sens de son engagement.

L'évènement contribue de ce fait à transformer la structure des configurations dans lesquelles l'action collective se déploie en redéfinissant les normes auxquelles doivent se conformer les acteurs. C'est de ce point de vue à l'aune des évènements dans le pays d'origine qu'il convient aussi d'envisager la recomposition actuelle d'un certain nombre de collectifs issus de l'immigration. Ces derniers peuvent en effet se trouver incités à convertir les orientations initiales en nouveau format d'expression susceptible d'avoir une plus forte résonnance dans l'espace public, car directement en lien avec l'actualité médiatique. En s'ouvrant ainsi plus largement à des problématiques nouvelles sur des thématiques d'actualité, elles contribuent aussi à renouveler le socle des adhérents et, ce faisant, encouragent de nouveaux jeunes à adhérer au mouvement. L'évènement ne saurait toutefois être assimilé à une rupture : dans de nombreux cas, il apparait bien plus comme la poursuite d'un système de relations de jeunes engagés dans des organisations transnationales mais

16. Terme employé par Kaïs, 33 ans, militant dans une association tunisienne.

17. Idem. 
dans des conditions profondément transformées à travers lesquelles se recomposent et se renouent de nouvelles alliances.

\section{L'engagement transnational en réaction : subir local et agir dans l'espace glo- bal}

Si un évènement inattendu dans le pays d'origine peut contribuer à éveiller ou réactiver des désirs d'agir dans l'espace transnational à partir d'une offre militante, associative, politique, humanitaire présente dans l'espace local, les ressorts de l'engagement pour les jeunes issus de l'immigration ne sont pas à rechercher que dans l'exceptionnel, l'inédit ou le « fait historique ». Le quotidien, le quartier, l'ordinaire peuvent aussi comporter des situations qui incitent des jeunes descendants d'immigrés à réagir, plus qu'à agir, dans l'espace transnational comme moyen de réponse à des offenses, des actes de racisme, de discrimination subis dans l'espace local. À ce sujet, l'enquête Trajectoires et Origines $(\mathrm{TeO})$ réalisée conjointement par l'INED et l'INSEE permet d'interroger ce lien entre expérience du rejet (discrimination, racisme, stigmatisation), pratiques transnationales et sentiment d'appartenance ${ }^{18}$. Il ressort de cette enquête par questionnaire que le sentiment pour les descendants d'immigrés, davantage encore que pour les immigrés, d'avoir vécu des situations discriminatoires ou racistes est fortement corrélé à un niveau important de transnationalisme ${ }^{19}$, qualifié par les auteurs de l'étude comme « réactif » : c'est d'abord parce que certains descendants d'immigrés ne se sentent pas acceptés en France qu'ils orientent, en réaction, une partie de leurs activités vers d'autres horizons que le pays dans lequel ils résident. C'est donc cette corrélation statistique établie entre discrimination ressentie et transnationalisme qu'il nous semblait pertinent d'interroger plus en profondeur au travers des réflexions tenues par certains enquêtés. Un certain nombre d'entre eux explique en effet leur engagement transnational par le sentiment d'exclusion éprouvé au niveau local, éventuellement alimenté par des difficultés d'intégration d'ordre socio-économique. Pour Assim, 35 ans, salarié et fondateur d'une association humanitaire algérienne basée à Ivry, les nombreuses épreuves de la discrimination rencontrées d'abord au cours de sa scolarité, puis tout au long de son parcours professionnel, l'ont incité à choisir entre trois options : «le

18. Plus spécifiquement sur la question du lien entre sentiment de discrimination et pratiques transnationales, voir Beauchemin C., Lagrange H., Safi M., « Liens transnationaux et intégration : entre ici et là-bas ", in Beauchemin C., Hamel C., Simon P., Trajectoires et origines. Enquête sur la diversité des populations en France, op. cit., pp. 87-115.

19. Les relations transnationales sont regroupées dans l'enquête TeO en quatre grands domaines : économique (investissements économiques hors métropole, propriété d'un bien hors métropole), politique (intérêt déclaré pour la politique dans le pays d'origine, appartenance à une association d'originaires), social (contacts personnels hors métropole, séjours dans la région d'origine, usages des médias dans la région d'origine) et symbolique (souhait de partir vivre hors métropole, souhait d'être enterré hors métropole). Le degré d'investissement transnational des individus est ainsi mesuré à partir d'un indicateur synthétique couvrant l'ensemble de ces domaines. 
silence, la colère, l'engagement pour une cause noble 20 ». S'il admet que c'est d'abord la colère qui a pris le devant face aux nombreux propos racistes ou gestes insultants tenus à son égard, son changement d'attitude s'explique par sa volonté de «montrer une image qui déplaît le plus aux personnes racistes : celle d'une personne issue de l'immigration qui réussit sans renier ses origines ». De là son souhait de créer une association humanitaire tournée vers le développement rural de l'Algérie et visant à faciliter l'accès des jeunes issus de l’immigration algérienne à des séjours humanitaires sur place, tout en leur permettant de " comprendre leurs origines, leurs racines, leur histoire, pour mieux la valoriser et la faire reconnaître à ceux qui la dévalorisent » (Assim, 35 ans, Algérie).

De la même manière que la situation décrite par Assim, certains jeunes descendants d'immigrés deviennent actifs sur le plan transnational à la suite d'une stratégie individuelle destinée à faire face à la discrimination et aux difficultés d'insertion auxquelles ils sont confrontés. Conscients du fait que le manque de qualification pourrait nuire à leur stabilité professionnelle, certains anticipent le danger potentiel et s'efforcent de devenir actifs dans le domaine transnational en vue d'accentuer des ressources, notamment linguistiques, culturelles ou sociales. Le champ transnational apparaît dans ce cas à la fois comme le lieu de reconnaissance mutuelle et de solidarité entre pairs, mais aussi comme le point de passage d'une «identité blessée 21 » vers un nouvel horizon des possibles. Il s'agit alors pour ces jeunes de « garder la face 22 » en affirmant défendre une citoyenneté autre que française, une appartenance qui vient se positionner comme acte de résistance. Lorsque ce sentiment négatif devient un «moteur » d'action transnationale, les pratiques adoptées auront tendance à accentuer le processus de différenciation. L'un de nos enquêtés, Koffi, 24 ans, affirme à ce sujet que les sources de son engagement dans une association sénégalaise à Vitry seraient moins à rechercher dans des orientations humanistes que dans le besoin ressenti d'adhérer plus fortement aux traditions auxquelles il était fréquemment renvoyé. Descendant d'immigrés sénégalais dont les parents étaient relativement peu investis dans le domaine associatif, son engagement dans une association sénégalaise s'explique par une volonté d'auto-affirmation, non seulement face à ses parents alors réticents à ce type d'engagement, mais aussi vis-à-vis de « ceux qui lui imposaient ce qu'il devait être » :

20. Extrait d'entretien. Assim, 35 ans, militant dans une association humanitaire algérienne.

21. Pollak M., Une identité blessée. Etude de sociologie et d'histoire, Paris, Editions Métailié, 1993.

22. On peut définir le terme de face comme étant « la valeur sociale positive qu'une personne revendique effectivement à travers la ligne d'action que les autres supposent qu'elle a adoptée au cours d'un contact particulier» (Goffman E., La présentation de soi. La mise en scène de la vie quotidienne, Les Éditions de minuit, 1974, p. 9). 
"Combien de fois on m'a appelé “l'Africain”, ou le "Sénégalais" ? Plein de fois. Mes parents ont toujours tout fait pour que je sois bien intégré et m'ont très peu parlé du Sénégal à la maison, même s’ils participent deux fois par an à l'organisation d'une fête à Vitry. Mais quand à l'école, avec tes amis, au foot, partout on t'appelle le Sénégalais, alors tu cherches à savoir ce que c'est qu'être Sénégalais. (...) Et tu vis comme un Sénégalais. Tu manges comme un Sénégalais. Tu lis des trucs sénégalais. Mes parents n’ont pas bien compris ce que je faisais, mais à un moment ça te permet de voir un peu plus clair et d'aller là où on veut de toi, d'avoir des nouveaux amis avec qui tu peux parler et t'éloigner de ceux qui te rejettent ». Koffi, 24 ans, Sénégal

Selon ces descendants d'immigrés, cet engagement transnational « en réaction » se construirait de manière différente de celui de leurs parents. Nés en France, ils affirment ressentir les situations de discrimination de façon nettement plus violente que leurs pères ou mères immigrés, dont le sentiment d'injustice associé aux discriminations subies pouvait, selon eux, être moindre car davantage intériorisé du fait de leur statut d'étranger ${ }^{23}$. Pour Zakia, militante à Saint-Denis dans une organisation humanitaire centrafricaine et également engagée dans une association de lutte contre le racisme, le sentiment d'injustice entre immigrés et jeunes issus de l'immigration constituerait un important facteur de différenciation entre générations. Les membres de l'association, tous plus âgés et principalement immigrés, se distingueraient sur ce point assez nettement des jeunes issus de l'immigration qu'elle rencontre dans le mouvement de lutte contre le racisme :

«Ils ne se sont jamais dit : "Tiens, pourquoi ils ne me considèrent jamais de la même manière que les autres ? Pourquoi ils me regardent toujours de travers ? Pourquoi ils me demandent toujours mes papiers ?”... Moi j'ai grandi ici, je suis née ici, et ça me semble clairement inacceptable. Je suis Française, et qu'on le veuille ou non, je ne dois pas être traitée différemment. Et je me battrai toujours si je considère que certaines choses sont inacceptables. Alors là-dessus, oui, je suis assez différente de mes parents (...). Pour moi, ils sont comme tous leurs amis de l'association (...), ce qui compte, c'est le village, les puits qu'ils vont creuser, tout ça. Par contre, ce qu'ils subissent en France, le racisme, tout ça : rien du tout ! Ça ne les intéresse pas !».

Zakia, 20 ans, Centrafrique.

23. Nous reprenons ici le point de vue des descendants d'immigrés rencontrés au cours de l'enquête dont le sentiment de discrimination, jugé par ces jeunes plus violent que celui de leurs parents, mériterait d'être davantage mesuré et mis en perspective avec le sentiment de discrimination réellement éprouvé par les immigrés eux-mêmes. Les nombreuses marches des immigrés depuis les années 1970 témoignent en effet d'une forte mobilisation qui trouve également sa source dans les épreuves du racisme et de la discrimination subies en France. 
Selon Zakia, l'engagement de ses parents visait aussi davantage à garder des liens avec les proches restés au pays, en vue d'un éventuel retour ${ }^{24}$. Par ailleurs, dans un contexte où la loi française interdisait encore aux étrangers, jusqu'en 1981, de se constituer en association, ces regroupements cherchaient à reproduire une organisation sociale et des formes de solidarité héritées de la communauté d'origine pour le bénéfice des familles et des communautés villageoises 25 . Pour leurs enfants, l'éventualité de vivre dans un pays dans lequel ils n'ont bien souvent jamais vécu est quasiment inexistante. Les actions transnationales engagées ne visent pas à conserver des relations ou à en créer de nouvelles dans le pays d'origine des parents, mais bien souvent à consolider celles existantes en France, dans le quartier, avec des personnes de même origine qui subissent elles aussi un faisceau de discriminations dans le territoire de résidence.

\section{L'engagement transnational en configuration : subir et agir " glocal»}

Evitons tout malentendu sur l'aspect unidirectionnel de l'engagement. Il serait en effet trop réducteur de penser l'« engagement en réaction » comme le passage à sens unique d'une discrimination subie au niveau local vers de nouvelles modalités d'action au niveau transnational, tout comme il serait erroné d'envisager l'« engagement dans l'action » à partir d'évènements survenus sur des terrains internationaux vers la construction ou la consolidation de réseaux de solidarité dans les territoires locaux. Davantage qu'une dichotomie entre l'échelle locale et l'échelle globale que pourraient abusivement laisser supposer ces trajectoires d'un échelon vers un autre, nous souhaitons bien plus insister ici sur les systèmes d'interaction entre ces différents niveaux. De façon plus concrète, les enquêtés pensent rarement les modalités de leurs actions militantes en opposant le territoire d'origine et celui du quartier. Ils les réinsèrent bien plus dans un ensemble cohérent, ni global, ni local, mais « glocal », où se mêlent des dimensions propres au territoire de l'histoire familiale et celles liées au territoire de résidence 26 . Par ce néologisme de "glocal ", nous souhaitons donc souligner que le rapport entre le local et le global n'est jamais direct et qu'il passe toujours par des processus d'adaptation, d'interprétation, voire de

24. Sur ce point, A. Sayad a tout particulièrement montré comment l'installation en France des immigrés issus principalement des terres maghrébines s'était construite autour d'un mythe, collectivement entretenu, celui d'une présence nécessairement provisoire qui appelait le retour des concernés sur leur terre d'origine (Sayad A., L'immigration ou les paradoxes de l'altérité, Bruxelles, De Boek-Wesmael, coll. L’homme / L’étranger, préf. Pierre Bourdieu, 1991).

25. Sur le paradigme développementaliste introduit par les solidarités villageoises des travailleurs immigrés africains, voir Dedieu J.-P., La parole immigrée. Les migrants africains dans l'espace public en France (1960-1995), Paris, Klincksiek, 2012.

26. Le néologisme "glocal » conceptualisé par Robertson permet précisément de conjuguer le niveau local et le niveau global en insistant sur le fait que le rapport entre eux est toujours une dialectique entre le dedans et le dehors, entre les logiques planétaires ou internationales et les logiques internes, nationales, voire locales (Robertson R., Glocalisation: Time-Space and Homogeneity-Heterogeneity in Global Modernities, Londres, SAGE Publication, 1995). 
traduction. Les différentes activités d'engagement produites par les jeunes de notre enquête se situent précisément dans cette recherche de cohérence entre l'international et le quartier, dimension qu'il s'agit de renouer dans le cadre des mobilisations engagées. Pour Assim, dont nous avons pu constater la volonté d'associer une valorisation des origines et une résistance aux épreuves de discrimination, son engagement passe nécessairement par la mise en relation entre le niveau international et le niveau local :

« J'ai toujours comparé ma situation discriminante en France à la discrimination de populations dans le monde qui vivent la même chose mais à un niveau international. (...) En fait, il faut trouver le juste milieu entre le quartier et l'international et défendre les droits des populations que ce soit près de chez nous, dans nos immeubles, nos régions ou à l'international. Dans ce sens-là pour moi il n'y a plus de frontières ».

Assim, 35 ans, Algérie

La distance physique ne va pas forcément de pair avec un affaiblissement du sentiment d'appartenance. La localité, comme le rappelle très justement Arjun Appadurai, est avant tout une question de relation subjective et de contexte, plutôt que d'enracinement à un espace matériel 27. Sans pour autant diluer cette localité dans un imaginaire abstrait qui serait constitué exclusivement par le sentiment de familiarité et de proximité entretenu avec un territoire étranger, ce n’est toutefois plus le lieu de résidence qui définit à lui seul l'horizon d'action de ces jeunes, mais les relations qu'ils tissent, dans le cadre de leurs activités militantes, avec des terrains internationaux. Pour Tijane, fondateur à Aubervilliers d'une association qui organise durant l'été des campschantiers au Sénégal, c'est bien dans cette imbrication entre dimension internationale et dimension locale que peut se comprendre son initiative, un enchevêtrement particulier lui permettant ensuite de donner un nouveau sens aux actions engagées à l'échelle du quartier :

«L'international c'est la finalité, mais il faut qu'on ait des actions ici au niveau local, non seulement pour recruter des jeunes, pour leur parler de l'association, et pour leur dire qu'on a aussi des projets de solidarité et voir si ça les intéresse. Je dirais presque parfois que l'international c'est un prétexte pour rendre leur quotidien meilleur ici et les faire agir plus fortement ».

Tijane, 31 ans, Sénégal.

27. Alors que la localité semble constituer un sol solide, objectif sur lequel peuvent s'appuyer les conceptions primordialistes, Appaduraï montre qu'elle est en fait une relation sociale intrinsèquement fragile qui suppose un travail quotidien de préservation (Appauraï $\mathrm{A}$., Après le colonialisme. Les conséquences culturelles de la globalisation, Paris, Payot, 2001). 
Cette porosité des frontières entretenue entre l'échelon local et l'échelon international semble aussi faciliter le caractère cumulatif des sentiments d'appartenance entre le territoire d'origine et celui de résidence ${ }^{28}$. Les pratiques d'engagement transnational n'apparaissent de ce fait pas exclusives d'un sentiment d'appartenance à la France. Au contraire, ces pratiques peuvent trouver une certaine cohérence dans le cadre de parcours biographiques cosmopolites où différentes trajectoires géographiques se cumulent.

\section{Transmission intergénérationnelle et rapport aux origines}

Réinsérer ces différentes carrières militantes de descendants d'immigrés dans le temps long de l'histoire migratoire soulève une série d'interrogations nouvelles sur les pratiques de transmission de savoir-faire et de représentations ainsi que des pratiques de citoyenneté d'une génération immigrée à une autre issue de l'immigration. De nombreux travaux ont déjà révélé comment pouvaient se maintenir des liens affectifs et matériels des anciens immigrés avec leur pays d'origine et comment ces liens pouvaient être transmis aux générations suivantes ${ }^{29}$. Reliées à l'engagement, il s'agit plus spécifiquement ici de voir comment certaines actions orientées vers le pays d'origine sont vectrices de nouveaux systèmes d'échange entre générations d'immigrés et populations issues de l'immigration, mais aussi de relations particulières entre lieu de l'histoire familiale et lieu de vie et d'engagement. Ces interconnexions entre « là-bas » et « ici » contribuent à modifier le sens attribué à « l'origine », dans une dynamique de réinvention d'une mémoire collective. En contexte migratoire postcolonial, ce rapport au pays d'origine peut être le produit d'injonctions et d'assignations identitaires provenant de différents univers (parents, familles, associations, institutions, etc.), mais aussi devenir un élément de revendication, d'affiliation, de réappropriation multiple en fonction des lieux et des interlocuteurs avec lesquels ces descendants d'immigrés interagissent. Différents réseaux d'influence peuvent alors intervenir pour proposer une sorte de puzzle mémoriel où des éléments de la trajectoire familiale passée viennent s'imbriquer dans le cours des engagements présents afin de donner une cohérence salvatrice à ceux qui pourraient douter de leur bonne-foi. Le

28. Sur les appartenances transnationales des descendants d'immigrés, voir Beauchemin C., Lagrange H. et M. Safi, "Liens transnationaux et intégration : entre ici et là-bas ", in Beauchemin C., Hamel C., Simon P., Trajectoires et origines. Enquête sur la diversité des populations en France, op. cit., pp. 87-115; Simon P., Tiberj V., "Les registres de l'identité. Les immigrés et leurs descendants face à l'identité nationale ", Ined, Documents de travail, $\mathrm{n}^{\circ} 176$.

29. Cette transmission de la génération immigrée à une autre issue de l'immigration peut aussi être analysée, comme dans les travaux de Lila Belkacem, à partir des processus sociaux de construction des origines, c'est-à-dire, selon cette auteure, tout ce à quoi le terme " origine(s) » renvoie classiquement : des supposées cultures, traditions, valeurs, identités, mais également des lieux et des collectifs y étant associés. Voir à ce sujet : Belkacem L., L'"enfant perdu" et le "pays d'origine". Construction des origines et expériences migratoires de descendants d'immigrants ouest-africains en région parisienne, Thèse de sociologie, EHESS, 2013. 
schéma ci-dessous permet précisément d'identifier les différents facteurs intervenant dans le processus de transmission et de réappropriation de la mémoire des origines, qu'il s'agisse de l'environnement familial (que nous nommerons les "porteurs de mémoire ») ou des différents milieux fréquentés par ces jeunes hors de la famille et pouvant entrer en conformité ou en opposition avec certaines valeurs familiales (que nous nommerons « les passeurs d'histoire » et qui rassemble les groupes de pairs, les associations de quartiers, les groupements religieux notamment).

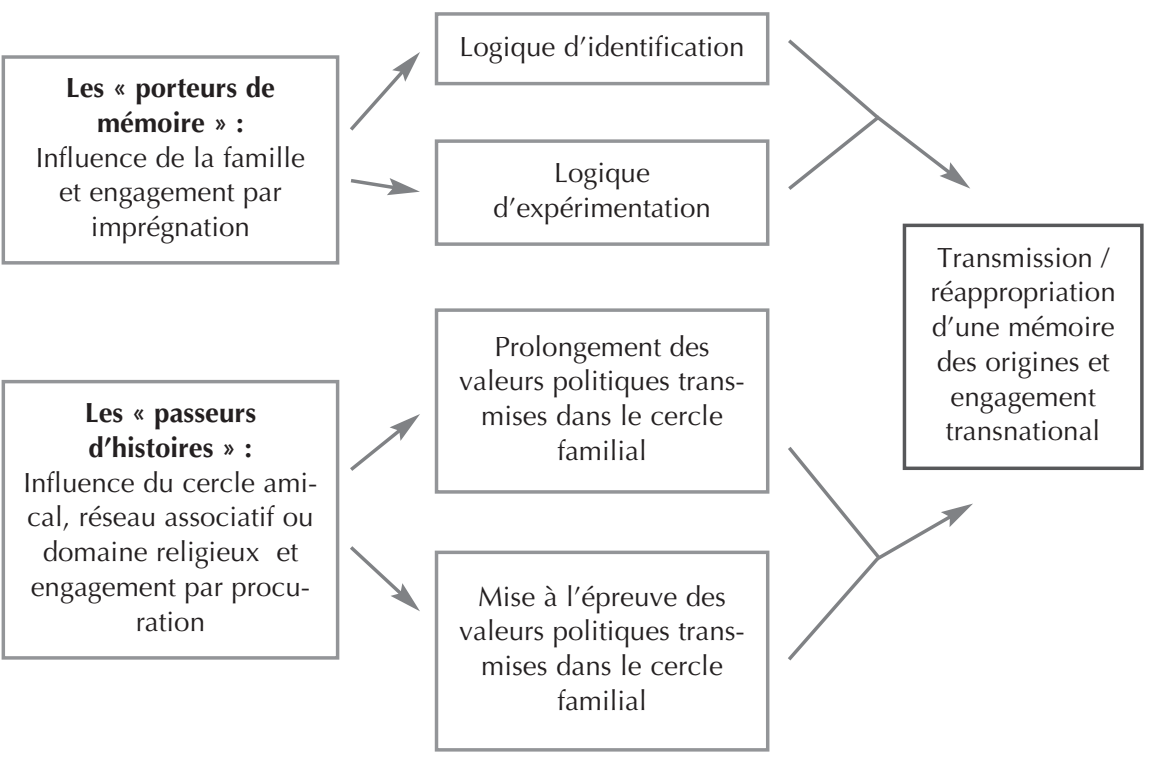

Graphique 2 : Schéma : engagement transnational des jeunes issus de l'immigration et réseaux d'influence

La famille et les "porteurs de mémoire » : logique d'identification et d'expérimentation

Un certain nombre d'auteurs s'accordent sur le rôle joué par le cadre familial dans la transmission d'un "fond de carte » à partir duquel pourront se construire de nouvelles modalités d'engagement ${ }^{30}$. Ce fond de carte est ensuite mis à l'épreuve des nouvelles expériences politiques rencontrées au fur et à mesure du parcours d'engagement. La reconstruction des carrières mili- 
tantes vise de ce point de vue à combiner « une analyse compréhensive des raisons d'agir avancées par les individus avec l'objectivation des positions successivement occupées par ces individus », qui travaille ensemble "les questions de prédispositions au militantisme, du passage à l'acte, des formes différenciées et variables dans le temps prises par l'engagement, de la multiplicité des engagements le long du cycle de vie (...) et de la rétractation ou extension des engagements 31 ». Différents éléments de relief pourront ainsi apparaître progressivement suivant deux logiques distinctes : une logique d'identification aux normes et valeurs transmises par la famille ; une logique d'expérimentation avec des jeunes qui font œuvre d'une certaine autonomie vis-à-vis des générations passées et d'innovation en matière de normes et de valeurs politiques.

Concernant la logique d'identification, il apparaît clairement au fil des entretiens que l'environnement familial joue bien sûr un rôle fondamental dans l'investissement associatif, politique ou humanitaire des jeunes rencontrés dans l'enquête, soit parce que les parents eux-mêmes sont - ou étaient engagés dans des collectifs ou des mouvements tournés vers le pays d'origine, soit parce qu'ils ont grandi dans un environnement familial autour duquel différents acteurs politiques ou militants associatifs pouvaient graviter. C'est notamment le cas de Sarrâ, 24 ans, engagée à Saint-Denis dans un mouvement de défense des droits de l'homme au Maroc et dont les parents avaient été fortement mobilisés dans des mouvements d'opposition avant leur arrivée en France. L'ensemble de son éducation a été imprégnée par cet engagement qu'elle qualifie de « total » et qui a largement dépassé la seule sphère familiale. Elle relate à ce sujet les actions contestataires engagées par son père pendant les années de plomb et qui lui ont valu une peine de huit années de détention: « Je suis née dans ce cadre-là, j’ai grandi avec ça, avec le militantisme (...) dans ce milieu-là où à la maison on débattait de droits de l'Homme, de liberté, des actualités, de ce qu'il se passe 32 ».

Au-delà des seuls motifs politiques évoqués pour expliquer le ralliement à une cause, il apparaît clairement que l'appartenance de l'un des parents, voire des deux, à un collectif et le fait de l'avoir fréquenté pendant l'enfance est un point d'accès non négligeable. L'univers familial apparaît comme un puissant moteur d'engagement transnational. Cet environnement transmet non seulement une réflexivité sur la situation politique dans le pays d'où sont issus les parents, mais aussi plus largement une identification subjective à un groupe d'engagés. Si ces carrières militantes ne se réduisent pas à une simple transmis-

30. Sur la socialisation politique au sein de la famille, voir Percheron A., La socialisation politique, Paris, Armand Colin, 1993 ; Muxel A., «La politisation par l’intime. Parler politique avec ses proches », Revue française de science politique, $\mathrm{n}^{\circ} 65,2015$, pp. 541-562.

31. Fillieule O., « Propositions pour une analyse processuelle de l'engagement individuel. Post scriptum ", Revue française de science politique, $\mathrm{n}^{\circ}$ 51, 2001, p. 202.

32. Sarrâ, 24 ans, Maroc. 
sion mécanique de génération à génération, on ne peut pour autant isoler le système de normes et de valeurs sociales en partie hérité auquel adhèrent ces jeunes adhérents ou militants ${ }^{33}$.

Pour des jeunes n'ayant jamais vécu dans le pays vers lequel ils orientent pourtant une part importante de leurs activités d'engagement, la famille apparaît comme un médiateur essentiel de la mémoire collective. L'environnement familial transmet une certaine lecture de l'histoire en fonction du système de représentations sociopolitiques qui lui est propre ou du rôle qui a pu être joué par ses membres au cours de certains événements dans le pays, conférant à ces derniers une réalité plus forte, plus proche mais aussi plus concrète. À l'image d'Hocine, fils d'opposant au pouvoir marocain et membre actif d'un mouvement contestataire, sa «fibre militante » lui viendrait selon lui de son « héritage familial » : "Mon père avait déjà été un militant d'extrême gauche au Maroc durant toutes les années 1970, jusqu'aux années 1990. Même s’il a essayé au maximum de ne pas m'engager dans son hobby, le fait d'avoir des journaux et des livres à la maison faisait que je devais obligatoirement m'intéresser à l'actualité et surtout à l'actualité politique 34 ». Son engagement transnational s'enracine dans la fidélité à la mémoire d'un membre de sa famille fortement investi dans une activité militante dans le pays d'origine. On se rapproche dans ce cas d'une forme de socialisation de l'engagement transnational par « imprégnation».

Mais il est aussi apparu au fil de l'enquête que la socialisation politique transmise au sein de la famille ne génère pas nécessairement, loin de là, des dispositions consonantes. On se retrouve dans ce cas non plus dans des logiques d'identification, mais d'expérimentation qui supposent des formes innovantes d'engagement éloignées des cadres de socialisation transmis par l'environnement familial. Dans ce cas de figure, les propos tenus par Salima, bénévole dans une association de soutien aux enfants maliens, révèlent des décalages importants entre certaines valeurs d'engagement transmises par la famille et celles développées ensuite par cette jeune adhérente. Au moment de la décision de Salima de rejoindre l'association malienne, des objections ont en effet rapidement été formulées par ses parents, eux-mêmes engagés dans des réseaux associatifs ou politiques français, mais qui conçoivent l'engagement transnational, plus encore lorsqu'il se rapporte au pays d'origine, comme ina-

33. Nous rejoignons sur ce point les travaux d'Elsa Lagier sur la transmission du rapport à l'engagement politique chez les enfants d'immigrés, plus particulièrement sur la question du processus de transmission et de réappropriation différenciée : "Cette transmission dépend aussi du choix effectué par les parents de transmettre ou non cette expérience à leurs enfants. Elle dépend enfin de la plus ou moins grande "réceptivité" des enfants devenus adultes, en fonction de la façon dont ils se représentent, ce qu'ils savent du chemin effectué par leurs parents, le message transmis étant toujours reconstruit par les destinataires » (Lagier E., «Les transmissions du rapport à l'engagement politique chez les enfants de migrants ", Migrations Société, nº123-124, 2009, p. 249.

34. Hocine, 30 ans, Maroc. 
dapté à une intégration " pleine et entière à la société française » :

«J'aimerais me tourner vers le management de projets internationaux. Mais ma mère n'y croit pas du tout, elle dit que c'est n'importe quoi que je fasse ça. Elle me dit de trouver dans le social. Ça entre dans tous les stéréotypes, elle dit que je suis "trop Africaine"... C'est un frein, ça me fait super mal quand on entre dans des stéréotypes pareils, surtout venant d'une mère (...). Je ne suis pas totalement dévouée à l'association, comme je l'aurais voulu, à cause de ce manque d'encouragement. Tout ce qui sort d'Europe, elle a un peu du mal ».

Salima, 23 ans, Mali

Le matériau d'enquête fait aussi apparaître dans plusieurs cas une conciliation de normes contradictoires entre d'un côté l'importance accordée par la famille à une assimilation qui passerait par une prise de distance avec la culture d'origine et, de l'autre, les tentatives de leurs enfants pour perpétuer cette mémoire dans des collectifs d'engagement. Face à la réticence de leurs parents, ces jeunes affirment une relative autonomie vis-à-vis d'eux et font également preuve d'innovation en matière de normes et de valeurs d'engagement. Surtout, ces situations démontrent que, contrairement à ce que l'on pourrait penser, la mémoire familiale et le lien au pays d'origine ne se transmettent pas nécessairement par «filiation» de parents à enfants, mais peuvent aussi se concevoir par "procuration» dans le cadre de nouvelles formes d'engagement où s'élabore et se reconstitue cette mémoire des origines. Dans ce cas, comme nous allons le voir, d'autres instances de socialisation peuvent jouer ce rôle de «passeurs d'histoire».

\section{Le cercle amical et les "passeurs d'histoire " : prolongement et mise à l'éprenve}

Aborder en termes de carrière l'engagement transnational des descendants d'immigrés suppose de s'intéresser non pas seulement à l'influence de l'univers familial, mais aussi à l'hétérogénéité des cadres de socialisation extérieurs à la famille que rencontrent ces jeunes au cours de leur vie. Pourvoyeuses de compétences multiples et parfois incompatibles, les carrières militantes de ces descendants d'immigrés se construisent dans des environnements diversifiés au cœur desquels ils sont amenés à incorporer de nouveaux modèles d'action. La transmission par les parents de valeurs politiques, militantes et/ou humanistes peut alors soit trouver des prolongements soit, à l'inverse, être mise à l'épreuve dans le cadre de nouvelles rencontres.

Dans le premier cas, il est possible d'observer une certaine continuité de sens et de valeurs d'engagement entre des modèles d'action fournis par l'univers familial et leur objectivation par des « autrui significatifs 35 » incarnés par 
des proches de l'entourage qui peuvent exercer une influence forte dans la validation et l'actualisation des valeurs d'engagement et du rapport tissé avec le pays d'origine. De nombreux interlocuteurs évoquent à ce sujet le rôle joué par le réseau amical et l'influence qu'il a pu avoir sur leur parcours d'engagement. Ce cercle d'amis peut certes fournir une information permettant l'entrée dans un parcours d'engagement, mais il peut aussi donner un avis, suggérer une option, et surtout ouvrir une voie nouvelle non encore anticipée dans le projet. Une simple idée échangée ou l'exemple incarné par un proche d'une autre voie que la sienne, peut clairement faire " pencher la balance » vers une issue ou une autre, ou ouvrir de nouvelles perspectives d'engagement.

Dans le second cas, des évènements survenus dans la vie personnelle liés à un déménagement, un changement d'orientation scolaire ou l'entrée dans la vie professionnelle ou dans une vie conjugale impliquent des « ruptures biographiques » qui peuvent placer ces jeunes engagés en contact direct avec de nouveaux univers sociaux et politiques jusqu'alors inconnus ou ignorés. Ces individus peuvent alors combiner plusieurs répertoires sociaux issus de contextes de socialisation qu'ils ont traversés et des groupes divers auxquels ils appartiennent. C'est alors le rapport à l'engagement transnational qui peut se trouver transformé en profondeur. Par exemple, la rencontre du futur compagnon ou de la prochaine compagne, peut contribuer à infléchir des trajectoires qui auraient pu être différentes. Sajida, dont nous avons déjà pu constater précédemment son important investissement militant dans une association tunisienne à la suite des évènements du Printemps arabe, nous explique qu'il n'en a pas toujours été ainsi. Si la résonnance médiatique de cet évènement et l'émulation qui s'en est suivie à Saint-Denis ont joué un rôle dans ses nouvelles activités militantes, un virage important a été opéré à la suite de la rencontre avec son futur mari, au cours d'une période où elle se situait selon elle dans un « réflexe identitaire » suscité par les polémiques sur le voile islamique :

«Pendant la crise du voile, $j$ 'ai eu un réflexe identitaire et je me suis un peu repliée, je me suis concentrée sur mon identité religieuse, sans pour autant - je suis pratiquante, ça pourrait faire écho à ce qui se passe actuellement - sans me radicaliser, mais avec une sorte de crispation (...). Après, il a fallu aussi que mon mari m'intègre dans la société française via son propre réseau et ses propres relations. Autrement je pense que j'aurais vécu un peu en communauté. Et cet engagement (dans le collectif tunisien pour la défense des droits de l'homme) m'a permis de trouver cet apaisement ici, et de continuer à m'impliquer pour mon pays ».

Sajida, 35 ans, Maroc

35. Mead G., L'esprit, le soi et la société (traduit de l'anglais par Jean Gazenneuve, Eugène Kaelin et Georges Thibault), Paris, Presses universitaires de France, 1963. 
Dans d'autres cas, les orientations choisies trouvent ainsi une certaine cohérence entre vie privée et domaine d'action. Dans la mesure où le réseau personnel contribue à la fois à intensifier la circulation d'idées mais aussi à consolider leur ancrage dans des projets d'engagement, il favorise du même coup les interactions répétées entre l'individu et son environnement ${ }^{36}$. Ces interactions confirment dans tous les cas l'idée que certains proches ont une influence plus significative que d'autres en infléchissant plus directement les parcours d'engagement. Plus qu'un rôle de " porteur de mémoire » généralement incarné par des parents qui véhiculent, par «filiation », différents fragments de l'histoire familiale, ces réseaux amicaux, " passeurs d'histoire » interviennent plus directement dans le cours des engagements présents en se situant en rupture ou en prolongement de cet héritage afin d'en définir les nouvelles trames. Certains peuvent aussi choisir de rompre avec cette idéalisation de la mémoire des origines en rappelant que leurs parents, tout en ayant connu une épreuve migratoire, peuvent aussi être responsables d'erreurs ou de mauvais choix. Ces jeunes sont aussi ceux qui manifestent le plus de distance à l'égard des traditions et d'une culture des origines, refusant bien souvent toute assignation identitaire qui ne les reléguerait qu'au simple rang d'héritiers d'une mémoire passée, alors qu'eux-mêmes souhaitent davantage s'afficher comme les transmetteurs d'une histoire présente qui reste encore à écrire. À cette vision de l' « immigration-maintien » à laquelle ces descendants se trouvent régulièrement relégués, s'oppose un «transnationalisme-rupture » dans lequel les parcours d'engagement témoignent dans bien des cas d'une certaine émancipation et auto-affirmation vis-à-vis des figures parentales, jugées par d'autres comme inaltérables et incontestables, tout en initiant de nouveaux parcours à l'échelle internationale.

$* * *$

Aborder en termes de carrières militantes les parcours d'engagement transnationaux des descendants d'immigrés, c'est tenter de mettre en relation, plutôt qu'en opposition, des dimensions objectives de conditions de possibilité de l'engagement (structures associatives au niveau du quartier, contexte social et politique, évènements) avec des dimensions subjectives à travers lesquelles se retravaille la question du sentiment d'appartenance. À ce titre, l'engagement transnational ne peut être qu'un parcours sinueux, incertain, traversé par des périodes de retrait, d'essoufflement ou d'investissement plus actif pendant lesquelles se renégocient non seulement la relation avec les partenaires de l'action militante mais aussi plus largement les rapports entretenus

36. La formation de l'identité de la personne est en effet étroitement liée au processus de socialisation qui la nourrit, l'étoffe et l'oriente. Claude Dubar écrit à ce sujet : "C'est de l'équilibre et de l'union de ces deux faces du Soi, le “moi” ayant intériorisé “l'esprit” du groupe et le “je” me permettant de m'affirmer positivement dans le groupe, que dépendent la consolidation de l'identité sociale et donc l'achèvement du processus de socialisation ", Dubar C., La socialisation. Construction des identités sociales et professionnelles, Paris, Armand Colin, 1991, p. 97. 
avec l'histoire familiale et les origines, lesquelles se trouvent tour à tour revendiquées, questionnées, passées sous silence ou réinventées. Le rapport entretenu par ces jeunes descendants d'immigrés avec le pays d'origine de leurs parents dans le cadre de pratiques d'engagement inscrites tout à la fois au niveau local et transnational pose de ce fait la question du système de valeurs et de sens accordé à la notion de citoyenneté. Cette dernière semble dans le cas présent davantage se définir par un mode de comportement et une participation active à la vie de la société que par un statut juridique lié strictement - et exclusivement - à la nationalité.

Les descendants d'immigrés remplacent, par leurs activités militantes transnationales, l'exclusivité des allégeances par la cumulativité des appartenances. Et c'est précisément autour de ces nouveaux régimes de citoyenneté que des distinctions peuvent apparaître entre immigrés et jeunes issus de l'immigration, du fait notamment d'une forte disparité des déterminants de l'engagement entre ces deux générations, non seulement s'agissant des causes objectives - mais également des raisons - subjectives - de l'action collective. Ces causes et ces raisons peuvent être situées sur un continuum entre deux polarités opposant le « réel » et l'« imaginaire » : la réalité objective d'un pays dans lequel les générations immigrées ont vécu et le sentiment subjectif d'une double appartenance pour des jeunes issus de l'immigration qui retravaillent, à travers leur engagement, leur histoire familiale et composent une nouvelle vision du monde qui ne passe plus par une allégeance principale à l'Etatnation, mais crée de nouveaux espaces identitaires pluriels et résolument cosmopolites. 